\title{
Ion Spectroscopy: Where Did It Come From; Where Is It Now; and Where Is It Going?
}

\author{
Tomas Baer ${ }^{\mathrm{a}}$ and Robert C. Dunbar ${ }^{\mathrm{b}}$ \\ ${ }^{a}$ Department of Chemistry, University of North Carolina, Chapel Hill, North Carolina, USA \\ ${ }^{b}$ Department of Chemistry, Case Western Reserve University, Cleveland, Ohio, USA
}

\begin{abstract}
The ASMS conference on ion spectroscopy brought together at Asilomar on October 16-20, 2009 a large group of mass spectrometrists working in the area of ion spectroscopy. In this introduction to the field, we provide a brief history, its current state, and where it is going. Ion spectroscopy of intermediate size molecules began with photoelectron spectroscopy in the 1960s, while electronic spectroscopy of ions using the photodissociation "action spectroscopic" mode became active in the next decade. These approaches remained for many years the main source of information about ionization energies, electronic states, and electronic transitions of ions. In recent years, highresolution laser techniques coupled with pulsed field ionization and sample cooling in molecular beams have provided high precision ionization energies and vibrational frequencies of small to intermediate sized molecules, including a number of radicals. More recently, optical parametric oscillator (OPO) IR lasers and free electron lasers have been developed and employed to record the IR spectra of molecular ions in either molecular beams or ion traps. These results, in combination with theoretical ab initio molecular orbital (MO) methods, are providing unprecedented structural and energetic information about gas-phase ions. (J Am Soc Mass Spectrom 2010, 21, 681-693) ( 2010 Published by Elsevier Inc. on behalf of American Society for Mass Spectrometry
\end{abstract}

$\mathrm{T}$ There are at least three perspectives guiding research in molecular spectroscopy of ions, which have given rise to rather distinct research communities. First is the goal of finding the quantum energy levels (electronic, vibrational, rotational) with high accuracy and energy resolution. Benefiting from experimental convenience, a vast body of results of this sort has been accumulated from photoionization and photoelectron spectroscopy experiments where the initial sample is the neutral molecule corresponding to an ion of interest.

Second is the question of "what color" the ion is, that is to say, taking the ion itself as the sample, observing the wavelengths and transition intensities of the optical absorptions or emissions of the ion. These experiments are difficult, but have great interest for the study of ions found in nature, as for instance among the community of astronomers trying to sort out the telescope spectra corresponding to molecular species existing in extraterrestrial environments.

Third is the use of spectra as a window to the identification and structural characterization of ionic molecules formed from interesting precursors by any of a variety of ionization, ion-molecule reaction, or ionextraction techniques. Combined with mass spectrometry, this branch of ion spectroscopy has developed into

Address reprint requests to Dr. T. Baer, Department of Chemistry, University of North Carolina, Chapel Hill, NC 27599-3290, USA. E-mail: baer@ unc.edu a new structural approach comparable to MS/MS, thanks to its ability to distinguish small conformational or structural differences among iso-mass ions. These studies have been dominated by the use of "action spectroscopy" (multi-photon and ion tagging dissociation techniques) to provide convenient, although indirect, access to the spectra of interesting ions.

The three major parts of this Perspective address these three different points of view on ion spectroscopy.

\section{Part 1. Finding Ion Quantum States Starting from Neutral Precursors}

\section{A. Synchrotron-Based VUV Threshold Photoelectron Spectroscopy with Ion Coincidence}

Since the inception of photoelectron spectroscopy in the 1960s [1-3], a large body of work has been published over the years $[4,5]$. In this experiment, a HeI light source producing photons with an energy of about 21 $\mathrm{eV}$ is used to ionize a gaseous sample, and the distribution of ejected electron energies is analyzed by a dispersive electrostatic energy analyzer. The resulting ion spectrum from its ionization energy (IE) up to $21 \mathrm{eV}$ shows all of the one-electron transitions possible from the neutral ground state. This method has been adapted to the study of free radicals [6-8] by the use of pyrolysis sources. However, the information content of these studies is limited by the modest energy resolution (typically $25 \mathrm{meV}$ ) of the electron monochromator.
(C) 2010 Published by Elsevier Inc. on behalf of American Society for Mass Spectrometry. 1044-0305/10/\$32.00

doi:10.1016/j.jasms.2010.01.028
Published online February 1, 2010 Received December 21, 2009 Revised January 27, 2010 Accepted January 27, 2010 
The new generation of synchrotrons are designed to accommodate many small bunches of tightly focused electrons that pass through undulators from which a highly directed light beam emerges [9-12]. When these sources are coupled with a high-resolution monochromator, the photon flux can be $10^{12} \mathrm{~s}^{-1}$ with an $0.01 \%$ band width (1 $\mathrm{meV}$ at $10 \mathrm{eV}$ photon energy). The resolution can be increased by a factor of 20 with a corresponding reduction in the flux $\left(5 \times 10^{10} \mathrm{~s}^{-1}\right)$, which makes this light source quite competitive with table top vacuum UV laser sources (see section 1B) in resolution, but considerably less intense than the laser based sources. The main advantage of the synchrotrons is the ease of tuning the radiation, the ability to exchange flux for resolution, and their operation as free user facilities. Among the vacuum ultraviolet (VUV) beamlines are those at the Swiss Light Source (SLS), Soleil, located in Saclay, which is close to Paris, BESSY II in Berlin, Diamond, in Darsbury, England, The Advanced Light Source (ALS) at the Lawrence Berkeley Laboratory, and the Chinese Synchrotron located in Heffei, China. In most cases, the user can arrive with only a sample in hand.

The marriage of velocity focusing [13] of the electrons with high-resolution synchrotron sources is a particularly ideal approach to high-resolution threshold photoelectron spectroscopy (TPES) [11, 14-16]. In this experiment, electrons and ions are extracted from the ionization region with moderate electric fields (e.g., $20-100 \mathrm{~V} / \mathrm{cm}$ ) using no grids. With a proper aperture size and spacing of the electrodes, the electrons from a large source can be focused according to their initial velocity perpendicular to the extraction field. Thus, electrons with zero kinetic energy in the perpendicular direction are focused to a tiny spot in the middle of an imaging detector. Electrons with higher velocity are focused into rings whose radius is proportional to the electron's perpendicular velocity component. With appropriate treatment of the electron images, $[17,18]$ it is possible to obtain a TPES with a resolution limited by the photon resolution.

The power of threshold photoelectron spectroscopy is the ability to collect such high-resolution TPES in coincidence with ions of known mass. In this experiment, electrons and ions are extracted in opposite directions, and the arrival times of threshold electrons as well as the ions are recorded. It is thus possible to plot the total threshold electron signal, or those threshold electrons that are collected in delayed coincidence with a given mass ion. As long as the internal energy of a given ion does not exceed its dissociation limit, the spectrum corresponds to a mass analyzed TPES. This coincidence approach is extremely useful when dealing with unstable free radicals or other transient species produced in a discharge or pyrolysis source. It is also essential when cooling the sample in a supersonic molecular beam, which generates a variety of clusters along with the cooled sample of interest. A recent example shown by Bodi at the conference (which may be the first of its kind) is shown in Figure 1 for the case of methyl iodide cooled in a seeded Ar beam [19]. The dominant species in the total TPES (solid points) is ascribed to the cold $\mathrm{CH}_{3} \mathrm{I}$. However, when the vertical scale is expanded, numerous clusters show up as well, including $\mathrm{ArCH}_{3} \mathrm{I}^{+}, \mathrm{Ar}_{2} \mathrm{CH}_{3} \mathrm{I}^{+}$, and $\mathrm{Ar}_{3} \mathrm{CH}_{3} \mathrm{I}^{+}$, whose sharp peaks below the IE of methyl iodide are readily identified according to their mass in the coincidence experiment. In addition, a TPES peak associated with the dissociative ionization of $\mathrm{Ar}^{-} \mathrm{CH}_{3} \mathrm{I}$ dimers to produce $\mathrm{CH}_{3} \mathrm{I}^{+}+\mathrm{Ar}+\mathrm{e}^{-}$shows up at $9.56 \mathrm{eV}$. From these results, it is possible not only to determine the IE of $\mathrm{CH}_{3} \mathrm{I}$ but that of the dimer and trimer with $\mathrm{Ar}$, and the bond energies of $\mathrm{Ar}-\mathrm{CH}_{3} \mathrm{I}$ in both the neutral and ionic manifold.

A unique feature of the French synchrotron, Soleil, is its crossed undulators that permit the generation of circularly polarized light, which can oscillate between left and right in about $20 \mathrm{~s}$. However, soon this will be upgraded to a switching time of $0.2 \mathrm{~s}$. As shown by Powis and Nahon at the conference, this polarization switching ability has been used to collect UV and VUV circular dichroism spectra of optically active molecules $[20,21]$. These experiments are carried out at fixed UV or VUV photon energies while collecting angularly resolved photoelectron spectra on an electron imaging detector.

\section{B. High-Resolution Laser-Based Spectroscopies}

Laser-based methods have been devised to generate vacuum UV light by a variety of nonlinear optical methods [22-24]. One of the simplest experimental approaches is third harmonic generation in a mixture of Xe and Ar [25]. The third harmonic of the Nd:YAG laser generates photons at $355 \mathrm{~nm}$. When this light is focused into a Xe/Ar cell, three photons are converted into a single photon with a wavelength of $355 / 3 \mathrm{~nm}$, thus

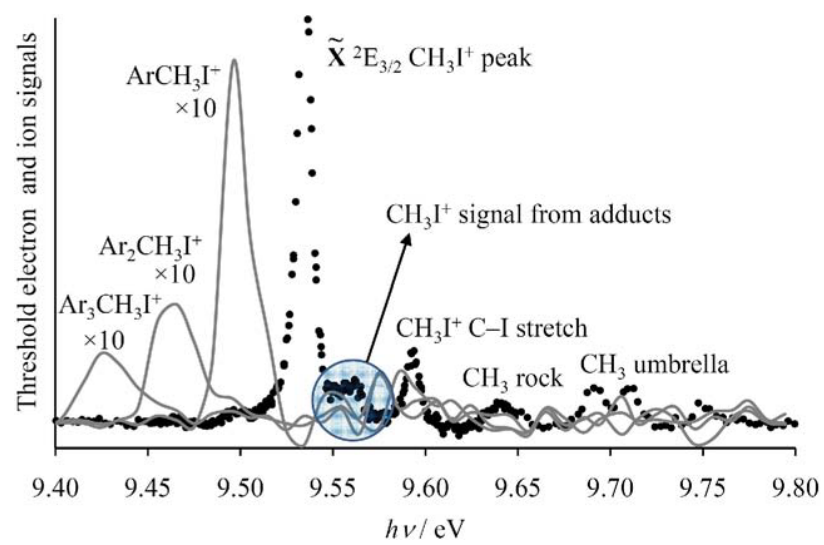

Figure 1. Threshold photoelectron spectrum of methyl iodide with the sample prepared in a molecular beam $\left(5 \% \mathrm{CH}_{3} \mathrm{I}, 95 \% \mathrm{Ar}\right)$. The points are the total TPES, while the solid grey lines are mass analyzed TPES. The ion mass associated with the small peak at $9.56 \mathrm{eV}$ is that of $\mathrm{CH}_{3} \mathrm{I}^{+}$, but its origin is the $\mathrm{CH}_{3} \mathrm{I}-\mathrm{Ar}$ dimer that has dissociatively ionized. 
generating light at $10.4 \mathrm{eV}$. Because it requires the simultaneous "absorption" of three photons, it is only possible with pulsed lasers that have a high peak power. The convenience of this process is due to the readily available third harmonic Nd:YAG photons and the location of the Xe absorption band near $10.4 \mathrm{eV}$. To obtain tunable VUV light, sum and difference frequency mixing schemes are utilized. A common scheme involves the 2-photon absorption of $212.5 \mathrm{~nm}$ light $\left(\mathrm{h} v_{1}=5.83 \mathrm{eV}\right)$ by $\mathrm{Kr}$ gas. A tunable laser $\left(\mathrm{h} v_{2}\right)$ is simultaneously focused in time and space to the same spot so that the $\mathrm{Kr}$ scatters light of energy $2 \mathrm{~h} v_{1} \pm \mathrm{h} v_{2}$. Various schemes have been developed that cover the VUV range from 5 to nearly $20 \mathrm{eV}$ with intensities about $10^{9}-10^{10}$ photons per second with a resolution of 0.2 $\mathrm{cm}^{-1}(.02 \mathrm{meV})$. The exceptional resolution coupled with a cold sample enhances the ionization efficiency because of the excellent overlap of the photon bandwidth and the natural line absorption line width. The signal to noise obtainable from these systems thus exceeds that for the synchrotron light sources. These light sources thus simultaneously exceed by factors of ten or more the synchrotron sources in both resolution and intensity. In addition, pulsed lasers permit the use of pulsed molecular beams, which, because of the high gas flux when the valve is open, are much more efficient in cooling the samples than continuous molecular beams. The major drawback of the VUV laser system is that the assembly of lasers and optical elements is complex and accessible only to laser experts, whereas the synchrotron sources are available to users who bring their samples to the installation. The total number of such broadly tunable VUV laser facilities is small, and probably about equal to the number of VUV beamlines at the various synchrotrons.

One photon ZEKE-PFI and MATI spectroscopy. The power of the VUV lasers is in their use in pulsed field ionization, PFI, (also called zero kinetic energy (ZEKE) spectroscopy) studies [26]. This approach is similar to the previously described threshold photoelectron spectroscopy except that in this case, the pulsed laser is used to excite Rydberg states lying just below an ion state. These long-lived neutral Rydberg states remain in the ionization region in the presence of a small (ca 0.5 $\mathrm{V} / \mathrm{cm}$ ) electric field, which is used to extract all of the prompt electrons that are associated with the production of lower energy ions. The prompt electrons are gone in a few microseconds after which an electric field sufficiently high to field ionize the Rydberg states is applied. These field ionized electrons are readily distinguished from the prompt electrons by their time of flight. Thus by scanning the laser wavelength while collecting the field ionized electrons, we obtain a very high-resolution $(<0.1 \mathrm{meV})$ photoelectron spectrum.

Many field ionization studies have been carried out with the absorption of one photon in the far UV or vacuum UV. Among these are a series of ZEKE/PFI studies on organometallic complexes with low ioniza- tion energies that permit the use of tunable UV lasers $[27,28]$. Recent results by the Yang group were presented at the conference. When the field ionized ions are collected rather than electrons, the process is termed mass analyzed threshold ionization (MATI) [29, 30]. The advantage of MATI is that the signal has the signature of the ion mass and thus can be used in a mixed sample. The group of $\mathrm{Ng}$ [31-33] used the ALS synchrotron to study the vibrationally, and at times rotationally resolved PFI spectra of nearly all stable diatomic and some triatomic molecules. In some cases, vibrational levels to nearly the dissociation limit were observed.

Multiphoton studies including UV-UV and IR-UV double resonance. Multiphoton studies are carried out by a two-color resonance enhanced multiphoton ionization (REMPI) scheme in which the first photon excites an intermediate vibrational or electronic state, and a second tunable photon is used to scan through the ion manifold of states. This approach permits selecting the neutral precursor state using the bound to bound absorption process of laser 1 . For instance, the trans and gauche rotational conformers of $n$-propylbenzene have been selected by resonant $X \rightarrow A$ transition using laser 1 , followed by tuning laser 2 through the ion states of each isomer [34]. Structural isomers of loosely bound dimers can also be selectively investigated [35]. That is, if a spectrum of a phenol-water dimer is desired, laser 1 can be use to select this dimer from a mixture of monomers, dimers, and trimers produced in a molecular beam [36]. In fact, it should even be possible to distinguish conformers and select a dimer in which the $\mathrm{H}_{2} \mathrm{O}$ is attached to the benzene ring or the $\mathrm{OH}$ group.

Another advantage of the two-color approach is the simplification of the photoelectron spectrum. This is particularly important in complex ions such as the allyl ion. It is difficult to produce an allyl radical in a discharge and to cool the radical sufficiently in a molecular beam to obtain a simple spectrum. Although $X i$ et al. [37] did just that and obtained a quite accurate ionization energy of this radical of $8.13146 \pm 0.00025$ $\mathrm{eV}$, the spectrum was not rotationally resolved and the IE could only be extracted by modeling the partially resolved first PFI-PES band. More recently, the Merkt group reported spectra that were much simpler and thus completely resolved when a particular rovibrational state in an excited electronic state was excited with laser 1, and the ZEKE/PFI spectrum was obtained by scanning laser 2 [38]. Their reported IE was $8.1309 \pm$ $0.0025 \mathrm{eV}$, which is $0.6 \mathrm{meV}$ lower and outside the experimental uncertainty of the two measurements. It might be argued that such a high precision $(0.25 \mathrm{meV}$ or $0.02 \mathrm{~kJ} / \mathrm{mol}$ ) is of little interest. However, without the high precision of these experiments, a complete assignment of all the peaks would have been impossible, and the assignment of the IE to better than $100 \mathrm{~cm}^{-1}(10$ $\mathrm{meV}$ ) would have been difficult. 
A final aspect of the two-color ZEKE/PFI experiment is the ability to access states not normally observed in a one-photon PES. The selection rule for photoionization is that only symmetric vibrational modes are excited. For instance, in the one color $\mathrm{CO}_{2} \mathrm{PES}$, only the symmetric stretch modes are excited, which show up beautifully in the A state $\mathrm{CO}_{2}^{+}$ion as a progression in the $\left(v_{i}, 0,0\right)(i=0,1,2, \ldots)$ ion vibrational modes. However, if an IR laser were to excite the asymmetric stretch $(0,0$, 1) mode in neutral $\mathrm{CO}_{2}$, then the resulting PES will exhibit the $\left(\mathrm{v}_{\mathrm{i}}, 0,1\right)(\mathrm{i}=0,1,2, \ldots)$ ion vibrational levels. In addition, if the IR laser excites a particular rotational state of the neutral molecule, and laser 2 is used to ionize this ro-vibrationally selected molecule, the spectrum is greatly simplified because very few rotational levels will be excited due to the predominance of the $\Delta \mathrm{J}= \pm 1$ selection rule. An example of these effects is shown in Figure 2 for the case of propyne [39]. In the upper two traces of Figure 2 are shown the 1 photon VUV photoionization efficiency scan and the pulsed field ionization photoelectron spectrum of propyne. The broad PFI-PES peak with a width of about $40 \mathrm{~cm}^{-1}(4$ $\mathrm{meV}$ ) is not resolved because of the many rotational transitions and because it consists of two electronic

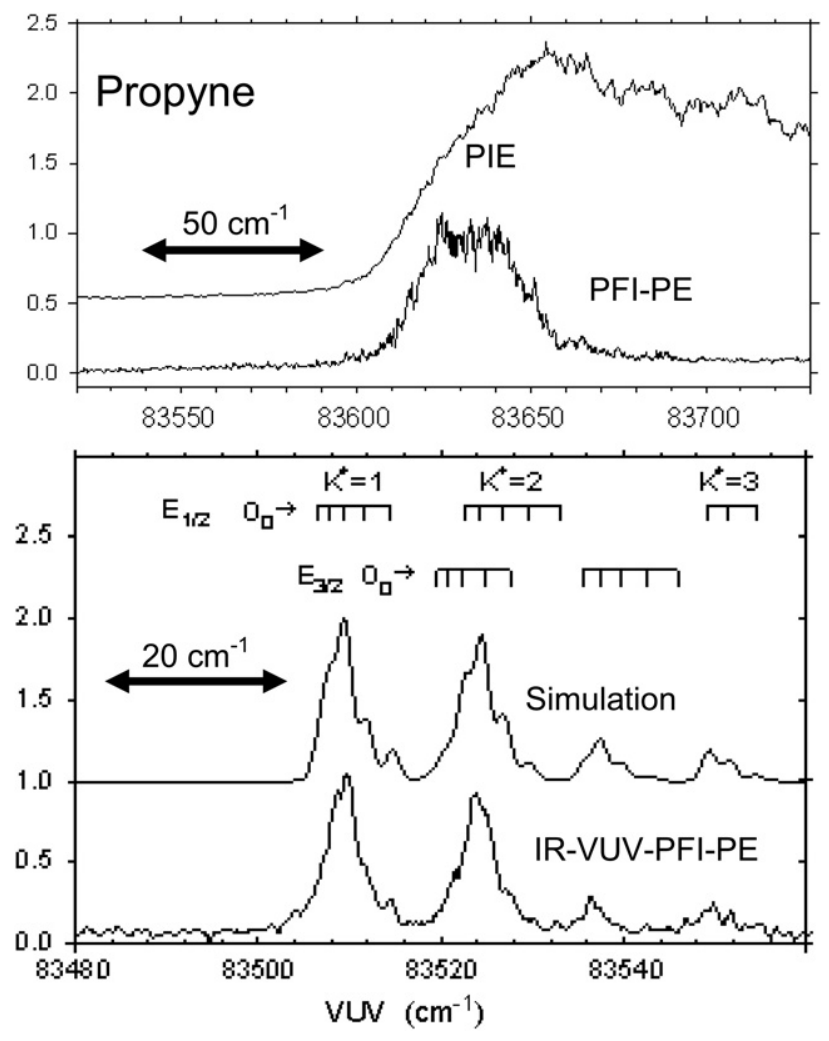

Figure 2. Upper traces are a one photon VUV photoionization efficiency (PIE) curve and a pulsed field ionization (PFI-PES) spectrum from the ground vibrational level of propyne to the ionic ground state of the ion. Below is a two-color version in which the $\mathrm{C}-\mathrm{H}$ stretch of propyne was excited before VUV photoionization. The transition is to the vibrationally excited state of the ion, which accounts for the shift in the wavelength between the one color and two-color experiments. Figure is adapted from Xing et al. [39] states that are split by spin-orbit interaction into the $E_{1 / 2}$ and $E_{3 / 2}$ states. Xing et al. [39] were able to simulate the spectrum, but an ionization energy based on a fully resolved spectrum would clearly be desirable.

The $\mathrm{Ng}$ group were able to collect a rotationally resolved PFI-PES of propyne using an IR photon to generate a rovibrational level in the neutral propyne, followed by a VUV photon with an energy less than the IE of propyne to produce the ion. By scanning the IR laser and keeping the VUV laser fixed, Xing et al. [39] mapped out the neutral IR spectrum, which exhibited well resolved $\mathrm{P}, \mathrm{R}$, and $\mathrm{Q}$ branches in the transition to the $v_{1}$ acetylenic $\mathrm{C}-\mathrm{H}$ transition at $3335.2 \mathrm{~cm}^{-1}$. By fixing the IR laser to one of these rovibrational transitions and scanning the VUV laser through the ionic manifold of states, a single rotational level in the $v$ level of the propyne molecule could be ionized. An example of such a spectrum is shown in the lower portion of Figure 2, in which the intermediate state was the P1 transition. The observed transition is from the ground $v_{1}$ level to the same $v_{1}$ level in the ion, whose value is $3217.1 \mathrm{~cm}^{-1}$, which is considerably lower than the neutral $\mathrm{C}-\mathrm{H}$ stretch frequency. The rotationally resolved spectrum in Figure 2 is particularly simple because the P1 origin is the $\mathrm{J}=0$ level of the vibrationally excited ground state of propyne. Higher rotational levels produced considerably more complex rotational structures in the ion spectra. These higher rotational levels in the neutral lead to more complex ionic spectra because the rotational selection rules for ionization are not as restrictive as they are in neutral bound to bound transitions $(\Delta \mathrm{J}=0, \pm 1)$. It is not unusual to observe $\Delta \mathrm{J}= \pm 5$ transitions in ionization because the electron can carry off some of the angular momentum. It is evident from the spectrum in Figure 2 that a complete rotational analysis is possible for the propyne ion and that its structure is well established. In addition, with this level of precision, the ionization energy of the propyne molecule is now established at $10.3674 \pm 0.0001 \mathrm{eV}$. These data deal only with the ground state of the propyne ion. However, such detailed analyses can clearly be carried out for a host of other vibrational bands of the propyne ion.

\section{Part 2. Direct Absorption and Emission Spectroscopies}

Studying absorption and emission of photons from ions of interest has been mostly restricted to visible and ultraviolet (UV/vis) wavelengths because infrared (IR) emission is very slow and thus weak, while direct detection of IR absorption is exceedingly difficult. Emission is generally easier to detect than absorption because the former is a zero background process, whereas absorption requires the measurement of the difference between large photon intensities. As a result, a wider variety of ions have been studied in fluorescence than in optical absorption. 
Emission from open shell ions (radical cations). Early in the growth of PES, it was observed that electronically excited (fluorescent) ions are created in the course of electron ejection from some favorable molecules, and emission studies of light from these radical cations became common in the 1970s and 80s [40-43]. The first low-quality spectra from photoionization and electron impact ionization were increasingly refined to give more information and higher resolution of vibrational structure, through development of techniques of coincidence of photon emission with photoelectrons, mass selection of ions, use of colder precursors to reduce thermal congestion, along with generally improved techniques.

It was found to be relatively unusual for organic radical cations to give high fluorescence quantum yields. The competing process of non-radiative decay to the electronic ground state usually dominates. However, quite a number of radical cations were found to give usably strong fluorescence emission as well as usefully resolvable vibrational fine structure. The literature of radical cation emission of small- to mediumsized organic molecules is dominated by those with substantial unsaturation giving fairly rigid molecular frameworks, leading to relatively low densities of vibrational states, and thus to relatively slow non-radiative decay processes. By 1990, it seemed that there could be few remaining organic molecules of reasonable volatility whose ions had not been examined for fluorescence emission, and a substantial literature of high quality spectra, as well as photoelectron-photon coincidence results, was available [41].

Emission from closed-shell ions. The situation is different for fluorescence study of closed-shell ions. Typically these might be protonated or deprotonated ions, formed by ion-molecule chemistry or by fragmentation, but these ion-formation processes do not commonly yield closed shell ions in excited, spontaneously fluorescent electronic states. Laser-induced fluorescence of trapped ions offers an alternative strategy, but heroic efforts in this direction had little success, and then only with radical cation species already well studied, such as the trifluorobenzene ion [44].

A new line of attack on this challenging goal was initiated by the demonstration by the Parks group [45] of usable laser-induced fluorescence of closed-shell dye molecule ions (e.g., rhodamines) introduced at high density into a quadrupole ion trap. This group and others, such as Jockusch's group, are expanding this new field into fluorescence study of gas-phase ions of interesting biomolecule systems. Parks and Jockusch were among the Conference speakers.

Direct absorption spectroscopy in plasmas. Observation of the wavelength-dependent light absorption of ions would be an ideal spectroscopic experiment, but sadly also among the most difficult approaches in the case of ions. The fundamental problem is the usually very low density of ions attainable, especially if the goal is a sample of mass-selected ions in the sample chamber. The challenge is thus that the fraction of incident light absorbed by the sample is exceedingly small.

The most favorable of the difficult approaches to this challenge is the use of plasmas at relatively high pressure. The ion density can be relatively large in these conditions, but the cost is that mass selection is not possible, and for all but very simple and favorable plasma chemistries, there is an intractable mixture of different ionic species. Two ingenious experimental approaches, velocity modulation and cavity ringdown, have been exploited to measure the ion absorptions in plasmas.

In velocity modulation spectroscopy [46], an alternating electric field is applied in the longitudinal direction in the plasma. The resulting oscillating velocity of the ions along the laser path gives an oscillating Doppler shift, which can be demodulated to give a highly effective way of extracting the ion light-absorption signal from the massive background signal of (unmodulated) neutral species, which also absorb light. Many simple ions have been observed in this way, notably in the Saykally lab beginning in the 1980s with ions such as $\mathrm{N}_{2}^{+}$and $\mathrm{NH}_{4}^{+}[47,48]$.

In cavity ringdown spectroscopy (CRS) [49], the plasma sample sits in a highly reflective cavity in which the laser initially establishes a standing wave of light. A small fraction of the light is extracted to measure the decay of the light as a function of time. In the absence of an absorber, the decay of this standing wave (or light intensity) is determined only by the losses at the mirrors. However, the presence of an absorber gives rise to additional light losses, and the lifetime of the standing wave decreases. Thus, as the photon wavelength is scanned through the various absorption bands of the sample, the cavity decay rate varies. Because of its high sensitivity, CRS is ideal for dilute samples. However, as in the case of velocity modulation spectroscopy, CRS does not identify the absorbing species except through its measured optical spectrum. Good examples of ion spectra measured this way are the polycyclic aromatic hydrocarbon (PAH ) ions observed in surveys of possible interstellar ionic species $[50,51]$. A recent rotationally resolved spectrum of tetraacetylene, which is of astrophysical interest, is shown in Figure 3 [52].

\section{Part 3. Action Spectroscopy}

In the action spectroscopy mode, an easily detected photochemical event, usually photodissociation or electron photodetachment, sometimes a photon-enhanced ion-molecule reaction, serves as a surrogate signal of photon absorption. The power of this technique is that the spectra have zero background in that a signal is observed only when a photon absorption event has taken place. However, the converse is certainly not true, and many situations arise where photon absorption 


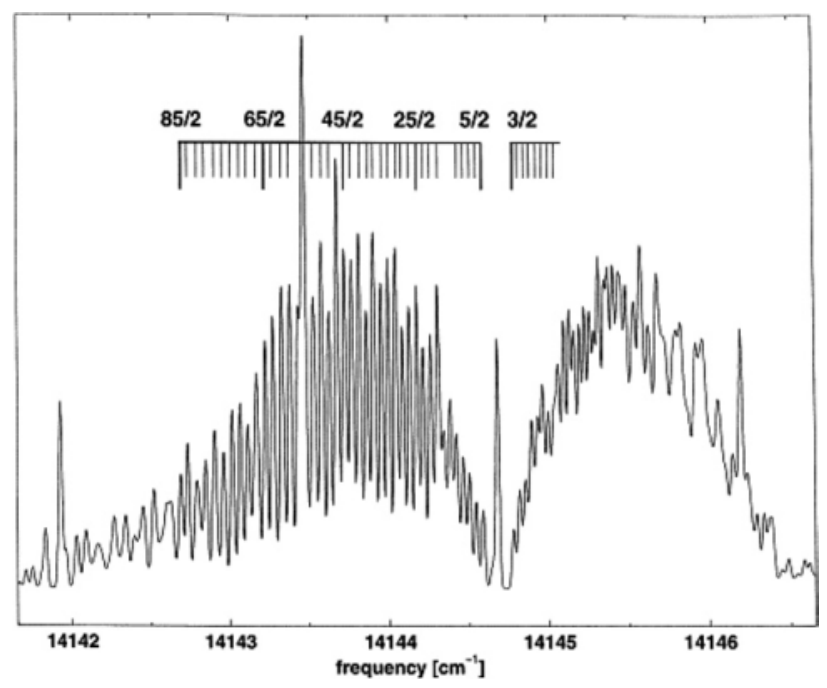

Figure 3. Rotationally resolved CRD absorption spectrum of the ${ }^{2} \Pi_{3 / 2} \leftarrow{ }^{2} \Pi_{3 / 2}$ electronic origin band transition of $\mathrm{HC}_{8} \mathrm{H}^{+}$, recorded through a supersonic planar plasma $\left(T_{\text {rot }} \approx 15 \mathrm{~K}\right)$. The rotational assignment is given. Some rotational lines were not observed because of overlap with $C_{2}$ transitions. Taken with permission from Pfluger et al. [52].

fails to be signaled by any observable photochemical event.

\section{A. UV/vis-Electronic Spectroscopy}

In the UV/vis region, absorption of a single photon is very often sufficient to induce subsequent dissociation. Dissociation is easily observed in a great variety of mass spectrometer and ion-beam instruments. Much electronic spectroscopy in this wavelength region was carried out in the 1970s and 80s with filter- or monochromatorselected light from lamp sources [53,54]. An example from van der Hart's lab [55] is shown here in Figure 4 in tribute to Wim van der Hart, one of the pioneers of ion spectroscopy, whose passing in 2009 was commemorated at the Conference. In this study, the photodissociation spectra of the parent cations of cyclohexane, $n$-hexane and 1-hexene, obtained by irradiating the samples with a monochromatized Xe arc source in an ICR cell, were compared between $250 \mathrm{~nm}(4.95 \mathrm{eV})$ and $700 \mathrm{~nm}(1.77 \mathrm{eV})$. Because the dissociation energy of all three ions to a variety of products [56] is well below 1.77 $\mathrm{eV}$, it was possible to obtain these spectra over the full visible range of the lamp. The different photodissociation spectra of the two isomers, cyclohexane and 1hexene, are clearly evident.

As tunable dye lasers became available, spectra with higher resolution began to be more common, an early example of which is shown in Figure 5, from one of the authors' laboratory.

A major part of the activity of the 1970s was the drift-tube work of the group at SRI led by J. T. Moseley on the spectroscopy and photochemistry of atmospheric cations and anions. A very successful drift-tube ion source, coupled with then-advanced tunable dye laser light sources, mapped out much of the UV/vis characteristics of most of the interesting ionic components of the upper atmosphere. Many spectra are unstructured, but some beautiful structured spectra were found, such as that of $\mathrm{CO}_{3}^{-}$across the visible, an early example of a polyatomic anion spectrum with extensive vibrational structure [58, 59].

Vibrational information about anions can also be obtained from high-resolution PES spectra of the anions themselves, where the kinetic energy spectrum of the detached electrons is measured using irradiation of the anion at fixed laser wavelength. For instance, the work of the Boulder group led by Ellison and Lineberger has provided numerous excellent examples of vibrationally resolved anion spectra and electron affinity determinations of such species as peroxy radicals [60]. An extensive review of experimental and theoretical work in this area has been published [61].

Notable in the development of UV/vis ion spectroscopy in this era was the work of the Freiser group. They pioneered the production, trapping, and photodissociation spectroscopy of metal-ion dimers, complexes, and clusters in the FT-ICR instrument. They worked hard on threshold photodissociation spectra for thermochemical information, but this application as a route to accurate thermochemistry has been almost entirely overtaken by the threshold CID approach, which has proven to have greater universality, precision, and reliability. The many photodissociation spectra of metal-containing ions (for example, Hettich et al. [62]) constitute a continuing background resource for modern work.

Moving toward more recent work exploiting better light sources in the UV/vis domain and advanced mass spectrometry, the photodissociation spectroscopy of larger $\mathrm{Fe}^{+2}$ organometallic complexes [63] by the Posey group attracted attention in the $90 \mathrm{~s}$, showing that gas-phase spectroscopy of organometallic ions was not restricted to the somewhat exotic complexes studied up to that time. Using electrospray they demonstrated the production and spectroscopy of complexes with oxidation states and ligands familiar to condensed-phase organometallic chemistry. Stace's group has been notable in carrying on the UV/vis action spectroscopy of such complexes, using a pickup molecular beam tech-

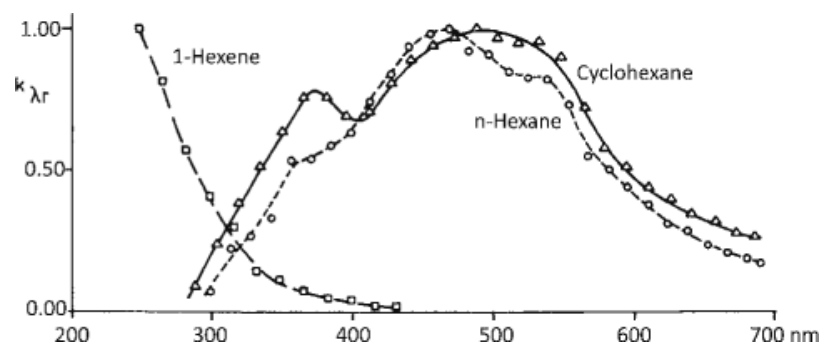

Figure 4. PDS spectra of two $C_{6}$ alkane ions are compared with that of 1-hexene using a Xe arc light source and ICR ion trapping and detection. Taken with permission from van Dishoeck [55]. 


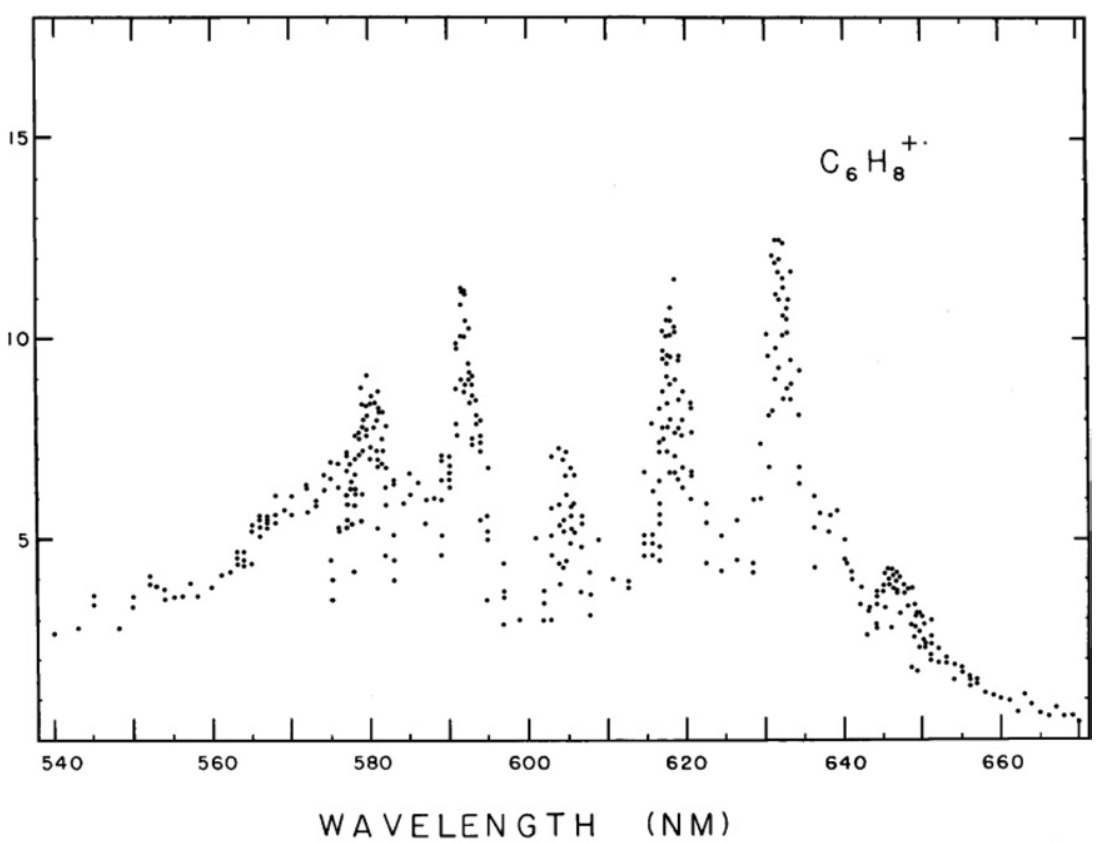

Figure 5. Photodissociation spectrum of $[1,3,5-\text { hexatriene }]^{+} \cdot$ showing vibrational information for two normal modes within the $\pi-\pi$ electronic excitation. Adapted from Dunbar and Teng [57].

nique to generate the ions. In such a source, a pulsed molecular beam of Ar picks up ablated ions generated by pulsed laser irradiation of a solid sample, thus cooling the ions in the supersonic expansion. A recent example [64] is the study of $\left[\mathrm{Zn} \text { (pyridine) }{ }_{4}\right]^{2+}$, showing extensive structure in the UV, which is challenging to assign. The Stace group's application of time-dependent DFT calculations to assist the assignment in this study illustrates the utility, but also the limited accuracy, of excited electronic state calculations. In fact, the quoted study left open the possibility that the observed spectral structure might be vibrational in its origin.

The Metz group has made notable advances in the modern era in applying action spectroscopy in the $\mathrm{UV} /$ vis regions to metal-containing ions of interest in catalysis [65]; see Metz' article in this issue.

\section{B. Infrared Spectroscopy of Ions}

It is in the area of infrared (vibrational) spectroscopy that the contemporary era has eclipsed earlier efforts. This dramatic burst of progress reflects maturation of two types of light source, the free electron laser and the optical parametric oscillator (OPO) laser. Also contributing has been the emergence of ion trapping mass spectrometers (most recently cryogenically cooled) and supersonic expansion molecular beam sources, along with electrospray ion production.

Current benchtop laboratory laser systems are based on an IR OPO operating at 10 to $20 \mathrm{~Hz}$, well matched to pulsed molecular beams. These lasers afford highresolution and increasingly useful light intensities. While the long-wavelength end of the spectrum is being steadily expanded with improving OPOs, uses of these sources are still largely confined to the blue end of the IR spectrum, from 2000 to $4000 \mathrm{~cm}^{-1}$. The other modern light source option is the IR free electron laser (FEL), which has higher mid-IR intensity and extremely broad tunability but considerably lower resolution (about $0.5 \%$ ). In the IR generating configuration, FELs are often operated in the mid-IR fingerprint region, from about 400 to $2200 \mathrm{~cm}^{-1}$, although expansion to either higher or lower wavelengths is possible. Two free electron lasers operating as user facilities are located at the FOM Institute in Nieuwegein (Utrecht, The Netherlands) and Orsay (Paris, France). The recent implementation of the free electron laser for intra-cavity experiment (FELICE) laser at the FOM, allowing intracavity sample operation in parallel with the lower power extracavity FELIX laser has raised the tunable CW power even higher, to the order of $10 \mathrm{~W}$. As has also been the case with synchrotron user facilities noted above, these FEL facilities have leveraged their extensive availability to non-expert outside users into extraordinary levels of scientific productivity.

Pioneering IR studies. Early work with inadequate technology gave only hints of the possibilities of IR spectroscopy in ion chemistry. Nearly all interesting photodissociation processes of untagged ions at IR wavelengths require multiple photons, which in general means that at least tens of milliwatts average laser power (or tens of millijoules per pulse in single-pulse experiments) are needed. This IR multiphoton dissociation (IRMPD) process using milliwatt laser powers is believed to proceed as follows. When the laser photon is resonant with an IR transition of some mode, $v_{i}$, a photon absorption event will excite the ion to $\mathrm{v}=1$ 
level of the ith mode. If the ion is reasonably large, rapid intramolecular vibrational relaxation (IVR) disperses this energy among several low-frequency modes. A second photon can thus excite the same $v_{i}$ mode again, followed by more IVR. [Transitions from $\mathrm{v}=1$ to 2 of the ith mode are thought to be less likely because anharmonicity will make these transitions less resonant. However, with sufficient laser power, such absorption events can become more probable.] After many such absorption/IVR steps, the ion will be heated sufficiently to cause dissociation. This IRMPD mechanism is often referred to as "ladder climbing." Using the available $\mathrm{CO}_{2}$ laser lines between 9 and $11 \mu$, some successful line spectra were achieved in ion-trapping (FT-ICR) instruments. Notable were the spectra of Shin and Beauchamp [66] of a few metal/ligand/nitrosyl complexes, which correlated well with condensed-phase spectra. In another direction, the distinction of isomeric organic ions using similar sparsely sampled spectra was successfully explored in work of the Eyler [67] and the Gaumann groups [68].

Several groups, including those of Eyler [67] and Lee [69], advanced the concept of combining a low-power tunable laser with a boost from a high-power fixedfrequency laser in a two-laser experiment: one photon of tunable IR, combined with a much larger increment of energy at a fixed wavelength. The trick is to set the intensity of the high power laser so that only ions that have absorbed the resonant photon will absorb the fixed-frequency laser and ultimately dissociate. These experiments gave a foretaste of two-laser schemes such as the IR/UV double resonance strategies being exploited in contemporary work as, for instance, in the Rizzo laboratory's work discussed below [70].

Other early successes pointing toward later directions were also achieved in ion-beam instruments, primarily using ion-tagging strategies. The results of Lee's group using $\mathrm{H}_{2}$ tagging action spectroscopy attracted attention [71], and can be seen as a milepost in the emergence of one-photon tagged-ion IR spectroscopy.

Ion tagging strategies. One-photon photodissociation is the preferred mode of action spectroscopy. However, one IR photon $(\sim 0.1 \mathrm{eV})$ is only sufficient to bring about dissociation in exceptional circumstances, including the case of intrinsically weakly bound ions (usually solventattached), or ions "solvated" or "tagged" with a weakly bound atom or molecule (usually $\mathrm{Ar}$ or $\mathrm{Ne}$, sometimes $\mathrm{H}_{2}, \mathrm{~N}_{2}$, etc.). Tagging ions requires ions colder than room temperature (usually below $150 \mathrm{~K}$ ) because the tagging atom will rapidly shake itself off from the ion by thermal motion at room temperature.

Spectral resolution in the IRMPD mode of spectroscopy is constrained by the linewidth of the laser, by band broadening due to thermal excitation of the sample, and also by the broadening effect of the multiphoton ladder climbing processes. As an example of the effect of multiphon broadening versus a one photon tagged-ion spectrum, Figure 6 compares the FEL spec-

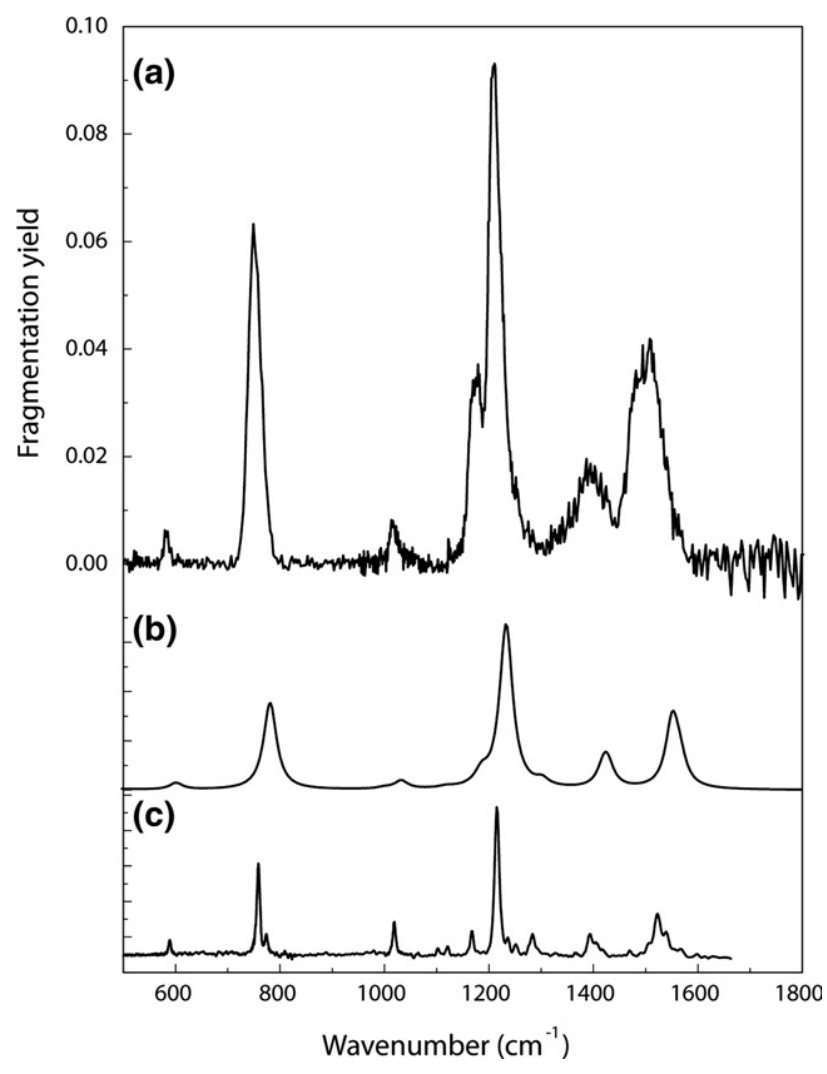

Figure 6. IRMPD spectrum (a), DFT computed spectrum (b), and Ar-tagged spectrum (c) of the naphthalene radical cation. Jetcooled ions were used in both experiments. The computed spectrum shows bands broadened with a Gaussian linewidth of 10 $\mathrm{cm}^{-1}$ FWHM. Taken with permission from Oomens et al. [73].

troscopy of naphthalene ion in the mid-IR obtained with IRMPD detection, and one-photon action spectrum of the Ar-tagged naphthalene ion obtained by Piest et al. [72]. Both spectra used jet-cooled ions, and both used the FEL as a laser source, so that thermal effects and laser-linewidth effects are similar. The Artagged one-photon spectrum is probably resolutionlimited by the FELIX laser width, whereas the IRMPD spectrum clearly shows the substantial additional broadening attributable to the multi-photon process.

Molecular beams of negative or positive ions from He-entrained laser ablation expansion from a discharge have been used in many studies, often with small ions, many of them containing metal ions. When metal ions from a laser-ablated metal intersect a molecular beam containing reactive gas, a host of species are created by ion-molecule reactions. In the course of the expansion these ions are cooled, making them ideal for spectroscopic interrogation. The ions can be stored in an ion trap for manipulation and mass selections, or they can be mass selected by their time of flight. An IR photodissociation laser can be directed along the path of the ions, through the ion trap, or (as in Duncan's experiment [74]), through the turning point in a reflectron where the ions are nearly stationary and easily photolyzed. 
(c)

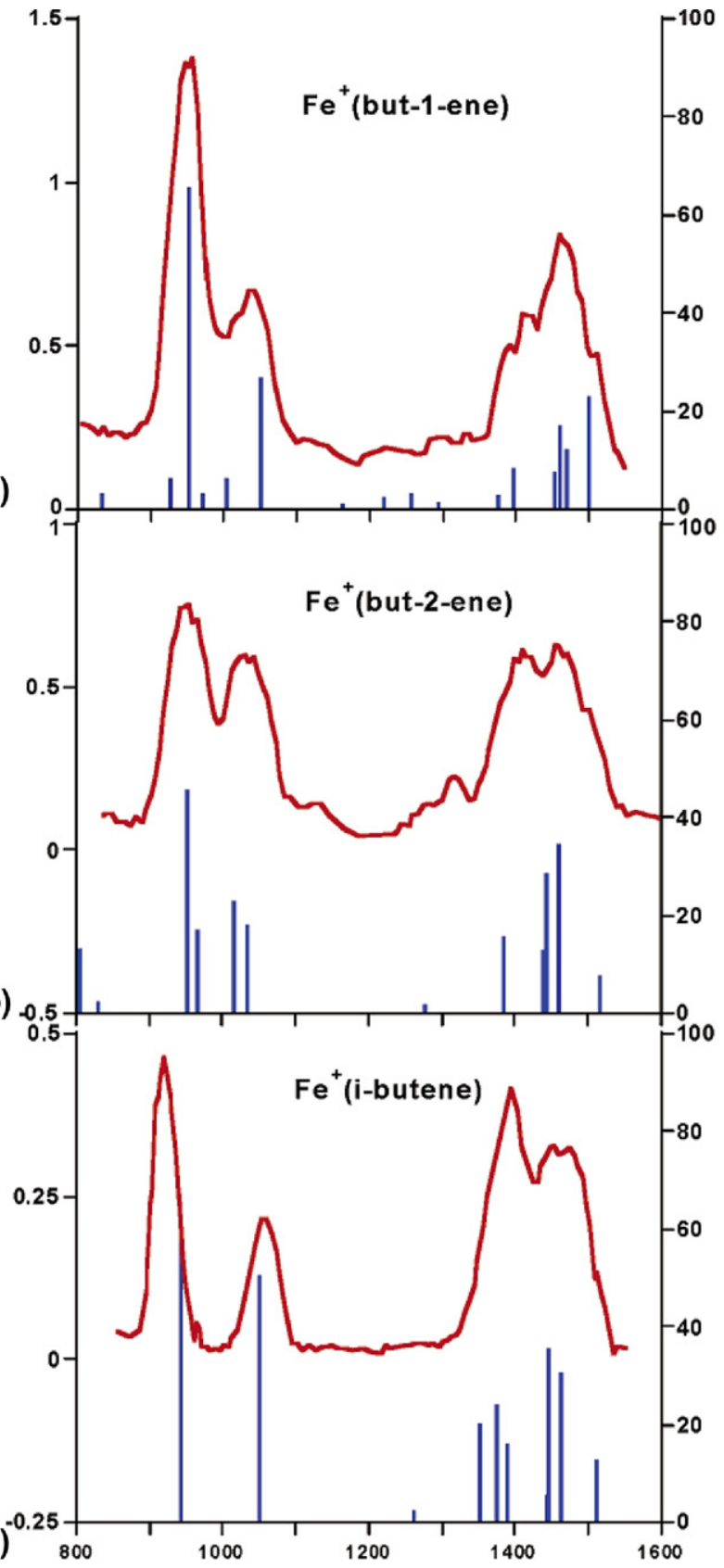

Figure 7. IRMPD and calculated IR absorption spectra (blue stick bars) of $\mathrm{Fe}(\text { i-butene })^{+}(\mathbf{a}), \mathrm{Fe}(2 \text {-butene })^{+}$(b), and $\mathrm{Fe}(1-$ butene $)^{+}(\mathrm{c})$. IRMPD yields (red line) and calculated intensities (right scale, $\mathrm{km} / \mathrm{mol}$ ). Taken with permission from Simon et al. [82].

By far the most highly resolved and most readily interpreted ion spectra have been obtained by laboratory based OPO lasers and Ar tagging. Among examples of closed shell ions studied in this manner are $\mathrm{H}_{5} \mathrm{O}_{2}^{+}[75,76], \mathrm{C}_{2} \mathrm{H}_{5}^{+}$[77], $\mathrm{C}_{2} \mathrm{H}_{3}^{+}$[78], as well as negative ions such as $\mathrm{CO}_{3}^{-}[79]$ and organometallic ions [74, 80]. The laser's very high spectral resolution and the cold ions in the argon expansion result in very sharp absorption peaks.
Multiple photon dissociation strategies with OPO and FEL lasers. The emergence of the FEL sources at Paris (CLIO) and at Utrecht (FELIX) signaled a surge of activity in mid-IR spectroscopy in the last decade. The capability of rapid, convenient scanning of the fingerprint IR spectrum amounted to a qualitative difference in the way IR was thought of as an ion characterization tool. These topics were well represented at the Conference, and in the present Special Issue with the papers of Maitre, Oomens, and Groenewold.

The publication of distinct isomeric $\mathrm{Fe} /$ hydrocarbon ion spectra from the CLIO lab $[81,82]$ (see Figure 7) sent a clear signal to the world about the power of this analytical tool, which continues at the FEL labs at a high level of activity. An early isomer distinction at FELIX was the demonstration of the ring-bound structure of the $\mathrm{Cr}^{+}$/aniline complex (Figure 8) [83], which showed that although the ring-bound and N-bound structures were computationally indistinguishable in energy, the experimental result gave a definitive answer. Increasingly, such applications of IRMPD spectroscopy in the IR are being complemented (often by the same groups) by scans across the $\mathrm{H}$-stretching region (2800-4000 $\mathrm{cm}^{-1}$ ) using benchtop OPO lasers (as displayed in Duncan's Conference presentation and contribution to the present issue).

The dream of applying gas-phase infrared spectroscopy to truly large biological systems was realized when the FELIX group reported a heroic experiment in which the IR spectrum of ionized cytochrome $c$ was observed [84]. This spectrum is displayed in Figure 9, along with spectra of potassiated complexes of simpler peptides (monopeptide and dipeptide) to show

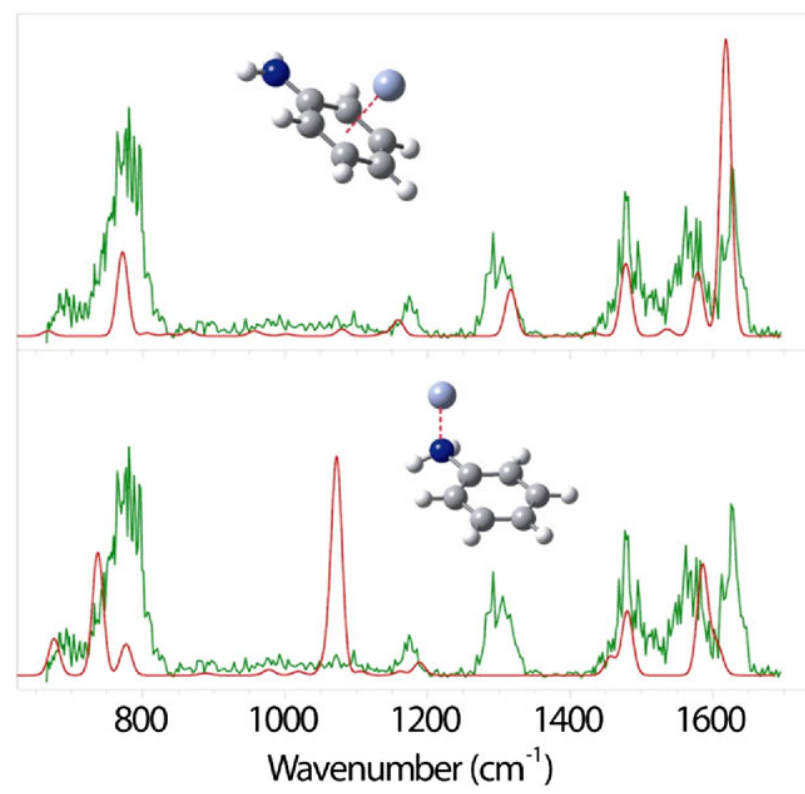

Figure 8. IRMPD spectrum of the $\mathrm{Cr}^{+}$/aniline complex with the experimental FELIX spectrum (green) compared with computed spectra (red) showing clear evidence of the ring-bound nature of the complexation. Adapted from Oomens et al. [83]. 


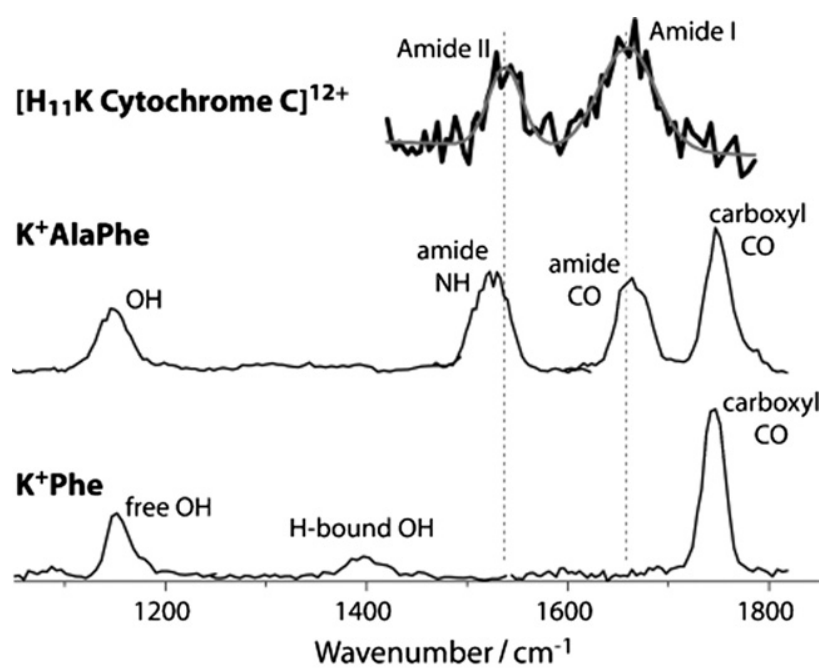

Figure 9. IRMPD spectra showing the evolution of peptiderelated IR bands going from potassiated amino acid (bottom) to the dipeptide (middle) to the large protein (c). Taken with permission from Oomens et al. [85].

the prominent features of the C-terminal group dominating the monopeptide case, the development of amide features in the dipeptide, and the complete take-over of the spectrum by the backbone amide groups in the protein [85].

Two-color UV/IR double resonance. A recent alternative to cooling ions in a molecular beam is the cooled ion trap that can store ions from an electrospray source at $10-20 \mathrm{~K}$ for use in spectroscopic experiments [86]. It is the ideal experiment for investigating large biomolecular ions because the cold ions yield sharp spectra, which are well matched to the OPO laser resolution. IR/UV optical double resonance methods can be utilized to
Family I

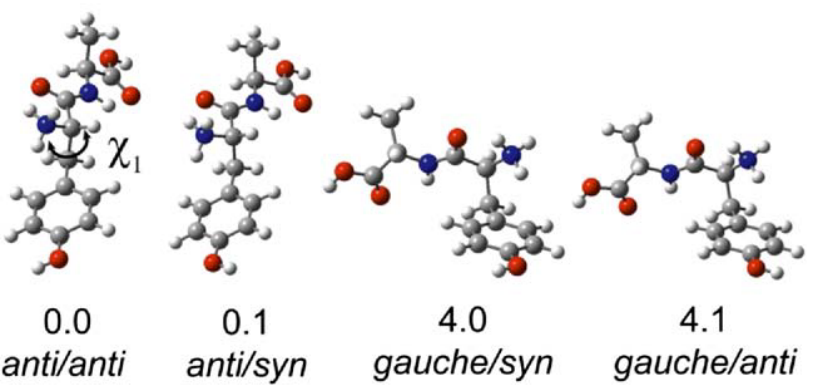

Figure 11. Structures and energies $(\mathrm{kJ} / \mathrm{mol})$ of $[\mathrm{H}-\mathrm{TyrAla}]^{+}$ ions calculated by B3LYP /6-311 $++\mathrm{G}^{* *}$ theory, which are identified with corresponding features in the UV/IR double resonance probe. The zero point energy corrected conformational energies are given in $\mathrm{kJ} / \mathrm{mol}$. Taken with permission from Stearns et al. [87].

determine the spectra of specific conformations among the many available to larger ions.

An example of this approach from the Rizzo lab is outlined in Figures 10, 11, and 12. The initial UV photofragment spectrum of protonated tyrosine-alanine dipeptide displayed in Figure 10 shows numerous absorption peaks that correspond to resonances in the UV at around $35,300 \mathrm{~cm}^{-1}$ [87]. Two spectra are shown, obtained by monitoring the ion intensities of two different photofragments ( $\mathrm{m} / \mathrm{z} 136$ and 146), and the observation of different UV absorption intensities in the two spectra confirms the presence of at least two conformations in the population. Calculations of various conformational structures shown in Figure 11 revealed four predicted conformations with sufficient stability to be significant components of the mixture. A two-color double resonance experiment was then used to obtain

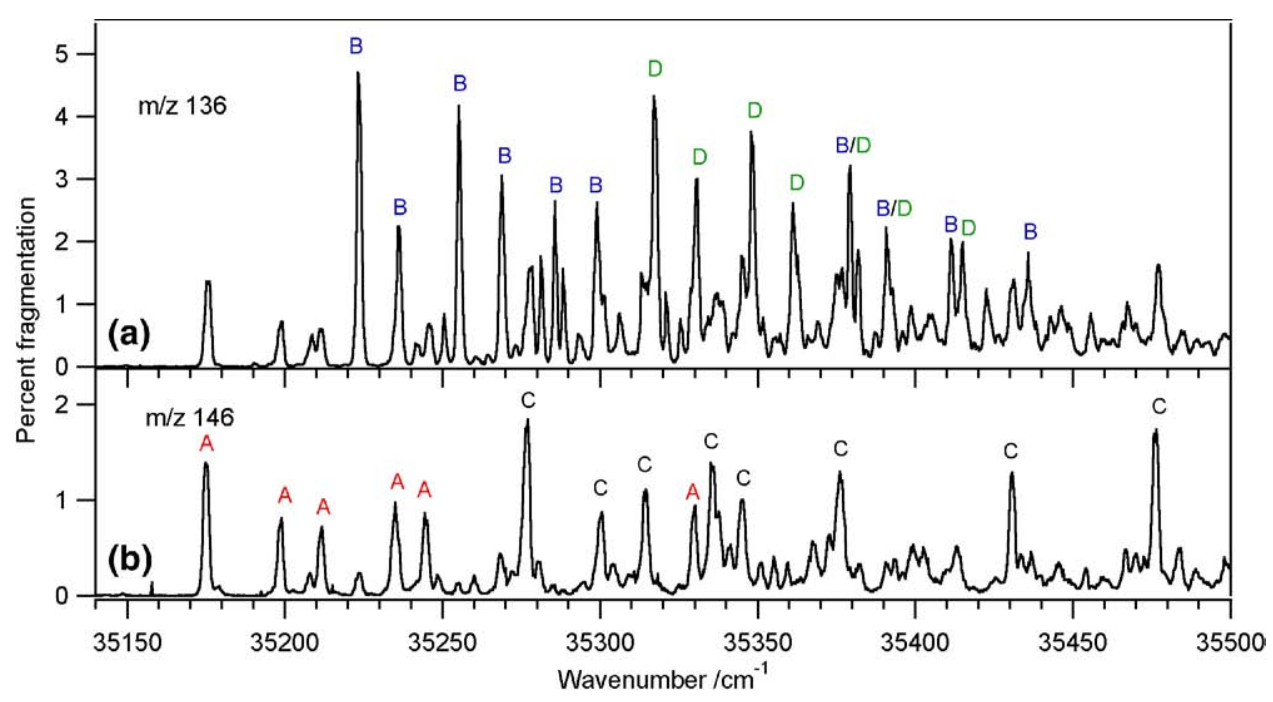

Figure 10. Ultraviolet photofragmentation spectra of $\mathrm{H}+$ TyrAla recorded by fragmentation into (a) mass channel 136 and (b) mass channel 146. The transitions are labeled by conformation, as determined by infrared-ultraviolet double resonance spectroscopy. Taken with permission from Stearns et al. [87]. 


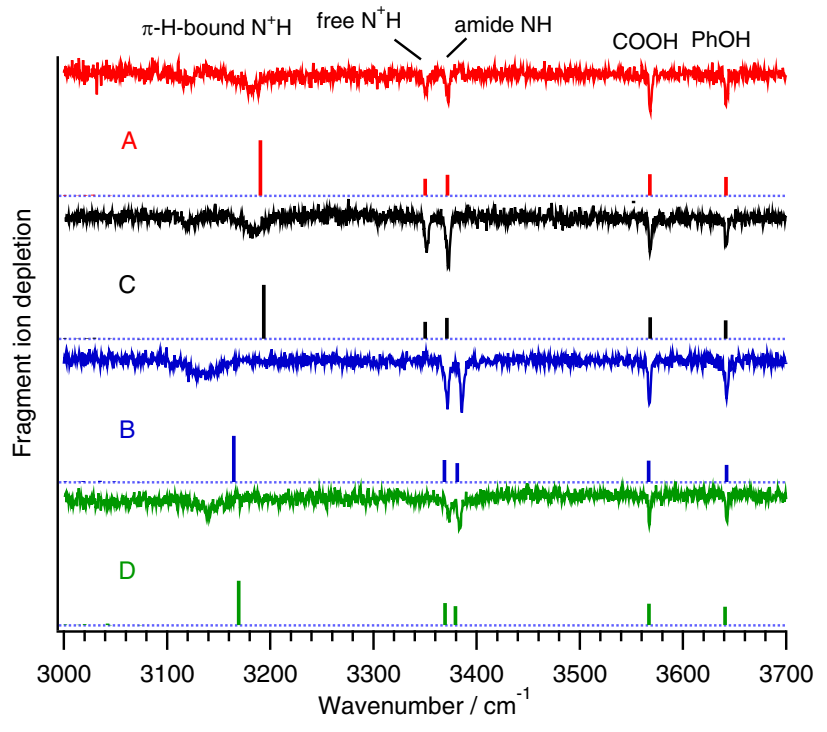

Figure 12. IR spectra of the four band origins of $\mathrm{H}^{+}$TyrAla conformations. The IR spectrum is obtained using a two-color, two-laser double-resonance method outlined in the text. The calculated stick spectra are shown for comparison. Taken with permission from Stearns et al. [87].

the IR spectra shown in Figure 12 of the four different isomeric ions, and to associate each of them with the corresponding calculated structure.

In the two-color experiment used to obtain the spectra in Figure 12, the UV laser was fixed on one of the peaks of the electronic spectrum associated with a particular conformation. A tunable IR laser pulse was fired some $100 \mathrm{~ns}$ earlier. When the IR laser is resonant with a vibrational transition in the ion, population is removed from the ground state of that conformation and the UV laser photofragmentation signal is thus depleted (an "ion dip"). Scanning the IR laser wavelength while monitoring the difference in the UV photofragmentation signal with the IR laser on and off gives the IR spectrum of each of the different conformations, as displayed in Figure 12. Finally, proceeding in this manner and using the IR spectra as fingerprints of the different conformations, all of the UV transitions that belong to a particular ion conformation can be identified (letters above the peaks in Figure 10). The various conformers are identified with the aid of calculated IR spectra, shown as sticks in Figure 12, as well as from the overall intensity of the spectrum, which should be related to the stability of the conformer.

Theory and computation. Advances in instrumentation have played a big role in the recent upsurge of interest in ion spectroscopy of larger molecular ions, but so also has the emergence of computational spectroscopy, particularly in the IR, as a strategy with sufficient accuracy and confidence to guide the interpretation of the flood of new observational results (see the paper of Ohanessian in the present issue for current developments in this area).
The calculated spectrum of naphthalene displayed in Figure 5, agreeing closely with the observed spectra, shows the high degree of fidelity of current DFT calculations of IR spectra. As is typical, the relative intensities agree less well with the IRMPD spectrum than with the one-photon spectrum, but the line positions are very satisfactory in both cases. Evidently, the Ar tag gives very little perturbation of the naphthalene ion mode frequencies in this favorable example. Calculation of excited electronic states has not yet reached this high a level of confidence for ions larger than very small ones, so theory has not yet achieved as high a degree of utility in the interpretation of UV/vis ion spectra.

\section{Conclusion}

The modern spectroscopy of gas-phase ions is advancing rapidly, especially in the areas of (1) extremely high-resolution state-resolved spectroscopic investigations of ionic molecules of reasonable size, (2) vibrationally and conformationally resolved spectroscopy of very large systems, particularly biomolecular ions, and (3) combining spectroscopy and calculations that lead to greater understanding of dynamical processes in ions. The Asilomar Conference showcased much of the active research encompassed within these areas. The present JASMS issue constitutes a valuable permanent resource arising from this event.

\section{References}

1. Turner, D. W.; Al-Joboury, M. I. Determination of Ionization Potentials by Photoelectron Energy Measurement. J. Chem. Phys. 1962, 37, 30073008

2. Turner, D. W.; Baker, C.; Baker, A. D.; Brundle, C. R. Molecular Photoelectron Spectroscopy; Wiley: New York, 1970.

3. Fahlman, A.; Hamrin, K.; Hedman, J.; Nordberg, R.; Nordling, C.; Siegbahn, K. Electron Spectroscopy and Chemical Binding. Nature 1966, $210,4-8$

4. Kimura, K.; Katsumata, S.; Achiba, Y.; Yamazaki, T.; Iwata, S. Handbook of He(I) Photoelectron Spectra of Fundamental Organic Molecules; Halsted Press: New York, 1981.

5. Rabalais, J. W. Principles of Ultaviolet Photoelectron Spectroscopy; John Wiley and Sons: New York, 1977.

6. Andrews, L.; Dyke, J. M.; Jonathan, N.; Keddar, N.; Morris, A. Photoelectron Spectroscopic Study of the Ground-States of $\mathrm{CH}_{2} \mathrm{Cl}^{+}, \mathrm{CHCl}_{2}{ }^{+}$, and $\mathrm{CHFCl}^{+}$. J. Am. Chem. Soc. 1984, 106, 299-303.

7. Butcher, V.; Costa, M. L.; Dyke, J. M.; Ellis, A. R.; Morris, A. A Study of the Phenyl Radical by Vacuum Ultraviolet Photoelectron Spectroscopy. Chem. Phys. 1987, 115, 261-267.

8. Sztáray, B.; Szepes, L.; Baer, T. Neutral Cobalt-Carbonyl Bond Energy by Combined Threshold Photoelectron Photoion Coincidence and $\mathrm{He}(\mathrm{I})$ Photoelectron Spectroscopy. J. Phys. Chem. A 2003, 107, 9486-9490.

9. Heimann, P.; Koike, K.; Hsu, C.-W.; Blank, D.; Yang, X. M.; Lu, K. T.; Ng, C. Y.; Evans, M.; Suits, A. G.; Flaim, C.; Lee, Y. T.; Padmore, H. A. Performance of the VUV High Resolution and High Flux Beamline for Chemical Dynamics Studies at the Advanced Light Source. Rev. Sci. Instrum. 1997, 68, 1945-1955.

10. Nahon, L.; Alcaraz, C.; Marlats, J. L.; Lagarde, B.; Polack, F.; Thissen, R.; Lepere, D.; Ito, K. Very High Spectral Resolution Obtained with SU5: A Vacuum Ultraviolet Undulator-Based Beamline at Super-ACO. Rev. Sci. Instrum. 2001, 72, 1320-1329.

11. Bodi, A.; Johnson, M.; Gerber, T.; Gengeliczki, Z.; Sztáray, B.; Baer, T. Imaging Photoelectron Photoion Coincidence Spectroscopy with Velocity Focusing Electron Optics. Rev. Sci. Instrum. 2009, 80, 034101/1$034101 / 7$.

12. Tang, X.; Zhou, X.; Niu, M.; Liu, S.; Sun, J.; Shan, X.; Liu, F.; Sheng, L. A Threshold Photoelectron-Photoion Coincidence Spectrometer with Double Velocity Imaging Using Synchrotron Radiation. Rev. Sci. Instrum. 2009, 80, 113101-1-113101/10.

13. Chandler, D. W.; Parker, D. H. Velocity Mapping of Multiphoton Excited Molecules. Adv. Photochem. 1999, 25, 59-106. 
14. Baer, T.; Li, Y. Threshold Photoelectron Spectroscopy with Velocity Focusing: An Ideal Match for Coincidence Studies. Int. J. Mass Spectrom. 2002, 219, 381-389.

15. Sztáray, B.; Baer, T. The Suppression of Hot Electrons in Threshold Photoelectron-Photoion Coincidence Spectroscopy Using Velocity Focusing Optics. Rev. Sci. Instrum. 2003, 74, 3763-3768.

16. Garcia, G. A.; Nahon, L.; Harding, C. J.; Mikajlo, E. A.; Powis, I. A Refocusing Modified Velocity Map Imaging Electron/Ion Spectrometer Adapted to Synchrotron Radiation Studies. Rev. Sci. Instrum. 2005, 76, 053302-1-053302/11.

17. Suits, A. G.; Continetti, R. E.; Eds. Imaging in Chemical Dynamics, American Chemical Society: Washington, DC, 2001

18. Whitaker, B. J. Image Reconstruction: The Abel Transform. In Imaging in Chemical Dynamics; Suits, A. G., Continetti, R. E., Eds.; American Chemical Society: Washington, DC: 2001; p. 68-86.

19. Bodi, A.; Shuman, N. S.; Baer, T. On the Ionization and Dissociative Photoionization of Iodomethane: A Definitive Experimental Enthalpy of Formation of $\mathrm{CH}_{3}$ I. Phys. Chem., Chem. Phys. 2009, 11, 11013-11021.

20. Powis, I.; Harding, C. J.; Garcia, G. A.; Nahon, L. A Valence Photoelectron Imaging Investigation of Chiral Asymmetry in the Photoionization of Fenchone and Camphor. Chem. Phys. Chem. 2008, 9, 475-483.

21. Garcia, G. A.; Nahon, L.; Harding, C. J.; Powis, I. Chiral Signatures in Angle-Resolved Valence Photoelectron Spectroscopy of Pure Glycidol Enantiomers. Phys. Chem., Chem. Phys. 2008, 10, 1628-1639.

22. Hepburn, J. W. Applications of Coherent Vacuum Ultraviolet to Photofragment and Photoionization Spectroscopy. In Vacuum Ultraviolet Photoionization and Photodissociation of Molecules and Clusters; $\mathrm{Ng}, \mathrm{C}$. Y., Ed.; World Scientific: Singapore, 1991; p. 435-486.

23. Seiler, R.; Paul, T.; Andrist, M.; Merkt, F. Generation of Programmable Near-Fourier-Transform-Limited Pulses of Narrow-Band Laser Radiation from the Near Infrared to the Vacuum Ultraviolet. Rev. Sci. Instrum. 2005, 76, 103103-1-103103/10.

24. Rupper, P.; Merkt, F. Intense Narrow-Bandwidth Extreme Ultraviolet Laser System Tunable up to 20 Ev. Rev. Sci. Instrum. 2004, 75, 613-622.

25. van Bramer, S. E.; Johnston, M. V. Tunable, Coherent Vacuum Ultraviolet Radiation for Photoionization Mass Spectrometry. Appl. Spectrosc. 1991, 46, 255-261.

26. Muller-Dethlefs, K.; Schlag, E. W. High Resolution Zero Kinetic Energy (ZEKE) Photoelectron Spectroscopy of Molecular Systems. Ann. Rev. Phys. Chem. 1991, 42, 109-136.

27. Lee, J. S.; Lei, Y.; Kumari, S.; Yang, D.-S. Metal Coordination Converts the Tub-Shaped Cyclo-Octatetraene into an Aromatic Molecule: Electronic States and Half-Sandwich Structures of Group III Metal-CycloOctatetraene Complexes. J. Chem. Phys. 2009, 131, 104304-1-104304/7.

28. Krasnokutski, S. A.; Yang, D.-S. High-Resolution Electron Spectroscopy and $\mathrm{s} / \mathrm{p}$ Structures of $\mathrm{M}$ (Pyridine) and $\mathrm{M}^{+}$(pyridine) $(\mathrm{M}=\mathrm{Li}, \mathrm{Ca}$, and Sc) Complexes. J. Chem. Phys. 2009, 130, 134313-1-134313/8.

29. Johnson, P. M.; Zhu, L. Mass Analyzed Threshold Ionization: Structural Information for a Mass Spectrum and Mass Information for Ionic Spectroscopy. Int. J. Mass Spectrom. Ion Processes 1994, 131, 193-209.

30. Bae, Y. J.; Kim, M. S. Photodissociation Spectroscopy of $\mathrm{CD}_{3} \mathrm{I}^{+}$Generated by Mass-Analyzed Threshold Ionization for Structure Determination. Chem. Phys. Chem. 2008, 9, 1709-1714.

31. Qian, X. M.; Lau, K. C.; He, G. Z.; Ng, C. Y.; Hochlaf, M. Vacuum Ultraviolet Pulsed Field Ionization Study of ND: Accurate Thermochemistry for the $\mathrm{ND}_{2}-\mathrm{ND}_{2}^{+}$and $\mathrm{ND}_{3}-\mathrm{ND}_{3}^{+}$System. J. Chem. Phys. 2004, $120,8476-8484$

32. Song, Y.; Ng, C. Y. Rotational-Resolved Pulsed Field IonizationPhotoelectron Study of $\mathrm{NO}^{+}\left(\mathrm{A}^{1} \sum_{-}, \mathrm{v}+=0-17\right)$ in the Energy Range of 17.70-20.10 eV. J. Chem. Phys. 2001, 115, 2101-2108.

33. Song, Y.; Evans, M.; Ng, C. Y. Hsu, C. W. Jarvis, G. K. Rotationally Resolved Pulsed Field Ionization Photoelectron Bands for $\mathrm{O}_{2}^{+}\left(\mathrm{a}^{4} \Pi_{\mathrm{u}}\right.$ $\mathrm{v}+=0-18)$ in the Energy Range of 16.0-18.0 Ev. J. Chem. Phys. 2000, $112,1306-1315$

34. Takahashi, M.; Kimura, K. Cation Vibrational Spectroscopy of Trans and Gauche n-Propylbenzene Rotational Isomers: Two-Color Threshold Photoelectron Study and Ab Initio Calculations. J. Chem. Phys. 1992, 97, 2920-2927.

35. Dessent, C. E. H.; Mueller-Dethlefs, K. Hydrogen-Bonding and van der Waals Complexes Studied by ZEKE and REMPI Spectroscopy. Chem. Rev. 2000, 100, 3999-4021.

36. Dopfer, O.; Muller-Dethlefs, K. $S_{1}$ Excitation and Zero Kinetic Energy Spectra of Partly Deuterated 1:1 Phenol-Water Complexes. J. Chem. Phys. 1994, 101, 8508-8516.

37. Xi, X.; Reed, B.; Lau, K.-C.; Ng, C. Y.; Zhang, X.; Ellison, G. B. Vacuum Ultraviolet Laser Pulsed Field Ionization-Photoelectron Study of Allyl Radical $\mathrm{CH}_{2} \mathrm{CHCH}_{2}$. J. Chem. Phys. 2007, 126, 171101-1-171101/4.

38. Gasser, M.; Schulenburg, A. M.; Kietiker, P. M.; Bach, A.; Merkt, F.; Chen, P. Single-Photon and Resonance-Enhanced Multiphoton Threshold Ionization of the Allyl Radical. J. Chem. Phys. 2009, 131, 014304-1014304/8.

39. Xing, X.; Bahng, M. K.; Reed, B.; Lam, C. S.; Lau, K.-C.; Ng, C. Y. Rovibrationally Selected and Resolved Pulsed Field IonizationPhotoelectron Study of Propyne: Ionization Energy and Spin-Orbit Interaction in Propyne Cation. J. Chem. Phys. 2008, 128, 094311-1-094311/4.

40. Horani, M.; Leach, S.; Rostas, J.; Berthier, G. Emission Spectrum of COS $^{+}$. J. Chem. Phys. 1966, 63, 1015-1025

41. Maier, J. P. Open-Shell Organic Cations: Spectroscopic Studies by Means of Their Radiative Decay in the Gas Phase. Acc. Chem. Res. 1982, $15,18-23$.
42. Maier, J. P.; Thommen, F. Relaxation Dynamics of Open-Shell Cations Studied by Photoelectron-Photon Coincidence Spectroscopy. In Gas Phase Ion Chemistry; Bowers, M. T., Ed.; Academic Press: Orlando, FL, 1984; p. 357-391

43. Maier, J. P. Spectroscopic Characterization of Mass-Selected Ions. Mass Spectrom. Rev. 1992, 11, 119-135.

44. Cage, B.; Friedrich, J.; Little, R. B.; Wang, Y. S.; McFarland, M. A. Hendrickson, C. L.; Dalal, N.; Marshall, A. G. Wavelength Resolved Laser-Induced Fluorescence Emission of $\mathrm{C}_{6} \mathrm{~F}_{3} \mathrm{H}_{3}^{+}$Trapped in an Ion Cyclotron Resonance Cell. Chem. Phys. Lett. 2004, 394, 188-193.

45. Iavarone, A. T.; Duft, D.; Parks, J. H. Shedding Light on Biomolecule Conformational Dynamics Using Fluorescence Measurements of Trapped Ions. J. Phys. Chem. A 2006, 110, 12714-12727.

46. Stephenson, S. K.; Saykally, R. J. Velocity Modulation Spectroscopy of Ions. Chem. Rev. 2005, 105, 3220-3234.

47. Polak, M.; Gruebele, M.; DeKock, B. W.; Saykally, R. J. Velocity Modulation Infrared Laser Spectroscopy of Molecular Ions: The $v_{4}$ Band of Ammonium. Mol. Phys. 1989, 66, 1193-1202

48. Saykally, R. J. Infrared Spectroscopy of Molecular Ions. Science 1988, 239, 157-161.

49. Linnartz, H. Cavity Ringdown Spectroscopy of Molecular Transients of Astrophysical Interest. In Cavity Ringdown Spectroscopy: Techniques and Applications; Berden, G.; Engeln, R., Eds.; Blackwell: Chichester, 2009; p. $145-180$.

50. Rouille, G.; Arold, M.; Staicu, A.; Krasnokutski, S.; Huisken, F.; Henning, Th.; Tan, X.; Salama, F. $\mathrm{S}_{1}\left({ }^{1} \mathrm{~A}_{1}\right) \leftarrow \mathrm{S} 0\left({ }^{1} \mathrm{~A}_{1}\right)$ Transition of Benzo[g,h,i]Perylene in Supersonic Jets and Rare Gas Matrices. J. Chem. Phys. 2007, 126, 174311-1-174311/11.

51. Tan, X.; Salama, F. Cavity Ringdown Spectroscopy of Jet-Cooled 1-Pyrenecarboxyaldehyde $\left(\mathrm{C}_{17} \mathrm{H}_{10} \mathrm{O}\right)$ and 1-Methylpyrene $\left(\mathrm{C}_{17} \mathrm{H}_{12}\right)$ Cations. Chem. Phys. Lett. 2006, 422, 518-521.

52. Pfluger, D.; Motylewski, T.; Linnartz, H.; Sinclair, W. E.; Maier, J. P. Rotationally Resolved A ${ }^{2} \Pi_{\mathrm{u}} \leftarrow \mathrm{X}^{2} \Pi_{\mathrm{g}}$ Electronic Spectrum of Tetraacetylene Cation. Chem. Phys. Lett. 2000, 329, 29-35.

53. Dunbar, R. C. Gas-Phase Ion Photodissociation. In Ionic Processes in the Gas Phase; Almoster Ferreira, M. A., Ed.; D. Reidel: Dordrecht, 1984; p. 179-203.

54. Dunbar, R. C. Ion Photodissociation. In Gas Phase Ion Chemistry, Vol. II Bowers, M. T., Ed.; Academic Press: New York, 1979; p. 182-220.

55. van Dishoeck, E. F.; Ewine, F.; van Velzen, P. N. T.; van der Hart, W. J. The Structures of Some Cycloalkane Molecular Ions by Photodissociation and Charge-Transfer Studies in an ICR Spectrometer. Chem. Phys. Lett. 1979, 62, 135-138.

56. Lias, S. G.; Bartmess, J. E.; Liebman, J. F.; Holmes, J. L.; Levin, R. D.; Mallard, W. G. NIST Chemistry WebBook. NIST Standard Reference Database Number 69; Mallard, W. G., Linstrom, P. J., Eds.; National Institute of Standards and Technology: Gaithersburg, MD, 2000.

57. Dunbar, R. C.; Teng, H. H. Photodissociation Spectroscopy of Hexatriene Cations. J. Am. Chem. Soc. 1978, 100, 2279-2283.

58. Cosby, P. C.; Moseley, J. T. Photodissociation Spectrum of Carbonate(-) Ion. Evidence for a Bound Excited State. Phys. Rev. Lett. 1975, 34 1603-1606.

59. Smith, G. P.; Lee, L. C.; Cosby, P. C.; Peterson, J. R.; Moseley, J. T. Photodissociation and Photodetachment of Molecular Negative Ions. V. Atmospheric Ions from 7000 to 8400 A. J. Chem. Phys. 1978, 68, 3818-3822.

60. Blanksby, S. J.; Ramond, T. M.; Davico, G. E.; Nimlos, M. R.; Kato, S.; Bierbaum, V. M.; Lineberger, W. C.; Ellison, G. B.; Okumura, M. Negative-Ion Photoelectron Spectroscopy, Gas-Phase Acidity, and Thermochemistry of the Peroxyl Radicals $\mathrm{CH}_{3} \mathrm{OO}$ and $\mathrm{CH}_{3} \mathrm{CH}_{2}$ OO. J. Am. Chem. Soc. 2001, 123, 9585-9596.

61. Rienstra-Kiracofe, J. C.; Tschumper, G. S.; Schaefer, H. F. I.; Nandim, S.; Ellison, G. B. Atomic and Molecular Electron Affinities: Photoelectron Experiments and Theoretical Computations. Chem. Rev. 2003, 102, 231-282.

62. Hettich, R. L.; Jackson, T. C. Stanko, E. M.; Freiser, B. S. Gas-Phase Photodissociation of Organometallic Ions. Bond Energy and Structure Determinations. J. Am. Chem. Soc. 1986, 108, 5086-5093.

63. Spence, T. G.; Trotter, B. T.; Burns, T. D.; Posey, L. A. Metal-to-Ligand Charge Transfer in the Gas-Phase Cluster Limit. J. Phys. Chem. A 1998, 102, 6101-6106.

64. Wu, G.; Norris, C.; Stewart, H.; Cox, H.; Stace, A. J. State-Resolved UV Photofragmentation Spectrum of the Metal Dication Complex $\left.[\mathrm{Zn} \text { (Pyridine) }]_{4}\right]^{2+}$. Chem. Commun. 2008, 4153-4155.

65. Citir, M.; Altinay, G.; Metz, R. B. Electronic and Vibrational Spectroscopy and Vibrationally Mediated Photodissociation of $\mathrm{V}^{+}(\mathrm{OCO})$. I. Phys. Chem. A 2006, 110, 5051-5057.

66. Shin, S. K.; Beauchamp, J. L. Identification of $\mathrm{Mn}(\mathrm{CO})_{\mathrm{n}} \mathrm{CF}_{3}^{-}(n=4,5)$ Structural Isomers by IR Multiphoton Dissociation, Collision-Induced Dissociation, and Specific Ligand Displacement Reactions: Studies of the Trifluoromethyl Migratory Decarbonylation Reaction in the Gas Phase. J. Am. Chem. Soc. 1990, 112, 2057-2066.

67. Peiris, D. M.; Cheeseman, M. A.; Ramanathan, R.; Eyler, J. R. Infrared Multiple Photon Dissociation Spectra of Gaseous Ions. J. Phys. Chem 1993, 97, 7839-7843.

68. Gaumann, T.; Riveros, J. M.; Zhu, Z. The Infrared MultiphotonDissociation Spectra of Bromopropene Isomeric Cations. Helv. Chim. Acta 1990, 73, 1215-1218.

69. Yeh, L. I.; Price, J. M.; Lee, Y. T. Infrared Spectroscopy of the Pentacoordinated Carbonium Ion $\mathrm{C}_{2} \mathrm{H}_{7}^{+}$. J. Am. Chem. Soc. 1989, 111, 5597-5604. 
70. Guidi, M.; Lorenz, U. J.; Papadopoulos, G.; Boyarkin, O. V.; Rizzo, T. R. Spectroscopy of Protonated Peptides Assisted by Infrared Multiple Photon Excitation. J. Phys. Chem. A 2009, 113, 797-799.

71. Okumura, M.; Yeh, L. I.; Myers, J. D.; Lee, Y. T. Infrared Spectra of the Cluster Ions Triaquahydrogen(1+)-Molecular Hydrogen $\left(\mathrm{H}_{7} \mathrm{O}_{3}^{+} \mathrm{H}_{2}\right)$ and Tetraaquahydrogen $(1+)$-Molecular Hydrogen $\left(\mathrm{H}_{9} \mathrm{O}_{4}^{+} \mathrm{H}_{2}\right)$. J. Chem. Phys. 1986, 85, 2328-2329.

72. Piest, H.; von Helden, G.; Meijer, G. Infrared Spectroscopy of Jet-Cooled Cationic Polyaromatic Hydrocarbons: Naphthalene ${ }^{+}$. Astrophys. J. 1999, 520, L75-L78.

73. Oomens, J.; van Roij, A. J. A.; Meijer, G.; von Helden, G. Gas-Phase Infrared Photodissociation Spectroscopy of Cationic Polyaromatic Hydrocarbons. Astrophys. J. 2000, 542, 404-410.

74. Velasquez, J.; Njegic, B.; Gordon, M. S.; Duncan, M. A. IR. Photodissociation Spectroscopy and Theory of $\mathrm{Au}^{+}(\mathrm{CO})_{\mathrm{n}}$ Complexes: Nonclassical Carbonyls in the Gas Phase. J. Phys. Chem. A 2008, 112, 1907-1913.

75. Diken, E. G.; Headrick, J. M.; Roscioli, J. P.; Bopp, J. C.; Johnson, M. A.; McCoy, A. B. Fundamental Excitations of the Shared Proton in the $\mathrm{H}_{3} \mathrm{O}_{2}^{-}$and $\mathrm{H}_{5} \mathrm{O}_{2}^{+}$Complexes. J. Phys. Chem. A 2005, 109, 14871490.

76. McCunn, L. R.; Roscioli, J. P.; Johnson, M. A.; McCoy, A. B. An H/D Isotopic Substitution Study of the $\mathrm{H}_{5} \mathrm{O}_{2}^{+} \mathrm{Ar}$ Vibrational Predissociation Spectra: Exploring the Putative Role of Fermi Resonances in the Bridging Proton Fundamentals. J. Phys. Chem. B 2008, 112, 321-327.

77. Ricks, A. M.; Douberly, G. E.; Schleyer, P. v. R.; Duncan, M. A. Infrared Spectroscopy of Protonated Ethylene: The Nature of Proton Binding in the Nonclassical Structure. Chem. Phys. Lett. 2009, 480, 17-20.

78. Douberly, G. E.; Ricks, A. M.; Ticknor, B. W.; McKee, W. C.; Schleyer, P. v. R.; Duncan, M. A. Infrared Photodissociation Spectroscopy of Protonated Acetylene and Its Clusters. J. Phys. Chem. A 2008, 112, 18971906.
79. Relph, R. A.; Bopp, J. C.; Johnson, M. A.; Viggiano, A. A. Argon Cluster-Mediated Isolation and Vibrational Spectra of Peroxy and Nominally $\mathrm{D}_{3 \mathrm{~h}}$ isomers of $\mathrm{CO}_{3}^{-}$and $\mathrm{NO}_{3}^{-}$. J. Chem. Phys. 2008, 129, 064305-1-064305/6

80. Duncan, M. A. Structures, Energetics, and Spectroscopy of Gas Phase Transition Metal Ion-Benzene Complexes. Int. J. Mass Spectrom. 2008, $272,99-118$.

81. Lemaire, J.; Boissel, P.; Heninger, M.; Mauclaire, G.; Bellec, G.; Mestdagh, H.; Simon, A.; LeCaer, S.; Ortega, J. M.; Glotin, F.; Maître, P. Gas Phase Infrared Spectroscopy of Selectively Prepared Ions. Phys. Rev. Lett. 2002, 89, 273002-1-273002/4.

82. Simon, A.; Jones, W.; Ortega, J. M.; Boisell, P.; Lemaire, J.; Maître, P. Infrared Multiphoton Dissociation Spectroscopy of Gas-Phase MassSelected Hydrocarbon-Fe ${ }^{+}$Complexes. J. Am. Chem. Soc. 2004, 126, 11666-11674.

83. Oomens, J.; Moore, D. T.; von Helden, G.; Meijer, G.; Dunbar, R. C. The Site of $\mathrm{Cr}^{+}$Attachment to Gas-Phase Aniline from Infrared Spectroscopy. J. Am. Chem. Soc. 2004, 126, 724-725.

84. Oomens, J.; Polfer, N.; Moore, D. T.; van der Meer, L.; Marshall, A. G. Eyler, J. R.; Meijer, G.; von Helden, G. Charge-State Resolved MidInfrared Spectroscopy of a Gas-Phase Protein. Phys. Chem., Chem. Phys. 2005, 7, 1345-1348.

85. Polfer, N. C.; Oomens, J.; Dunbar, R. C. Alkali Metal Complexes of the Dipeptides PheAla and AlaPhe: IRMPD Spectroscopy. Chem. Phys. Chem. 2008, 9, 579-589.

86. Rizzo, T. R.; Stearns, J. A.; Boyarkin, O. V. Spectroscopic Studies of Cold Gas-Phase Biomolecular Ions. Int. Rev. Phys. Chem. 2009, 28, 481-515.

87. Stearns, J. A.; Guidi, M.; Boyarkin, O. V.; Rizzo, T. R. ConformationSpecific Infrared and Ultraviolet Spectroscopy of Tyrosine-Based Protonated Dipeptides. J. Chem. Phys. 2007, 127, 154322-1-154322/7. 